\section{Cost analysis of integrated community case management of childhood malaria, diarrhea and pneumonia enhanced by mobile health technology in rural Zambia}

\author{
Godfrey Biemba, ${ }^{1,2}$ Arnold Mulenga, ${ }^{3}$ \\ Boniface Chiluba, ${ }^{4}$ Ulla K Griffiths, ${ }^{5}$ \\ Kojo Yeboah-Antwi, ${ }^{2}$ \\ William MacLeod, ${ }^{4}$ Karsten Lunze, ${ }^{2}$ \\ Davidson H. Hamer ${ }^{2,6}$ \\ ${ }^{1}$ National Health Research Authority, \\ Lusaka, Zambia; ${ }^{2}$ Department of Global \\ Health, Boston University School of \\ Public Health, Boston, MA, USA; \\ ${ }^{3}$ Ministry of Health, Lusaka, Zambia; \\ ${ }^{4}$ Zambia Centre for Applied Health \\ Research and Development, Lusaka, \\ Zambia; ${ }^{5}$ United Nations International \\ Children's Fund (UNICEF), New York, \\ USA; 'Section of Infectious Diseases, \\ Department of Medicine, Boston \\ Medical Center, Boston, MA, USA
}

\begin{abstract}
Integrated community case management (iCCM) of malaria, diarrhea, and pneumonia is a comprehensive, equitybased strategy to improve treatment access for underserved children under five years old. This paper presents data on cost of iCCM and incremental costs of mHealth enhanced supervision and supply chain management in Zambia. We collected cost data using three questionnaires applied at national, district, health facility and community levels. We interviewed 40 health facility supervisors and 75 community health workers. A provider perspective and an ingredient costing method was used. We entered and analyzed data in a customized excel costing tool. The result shows that total $\mathrm{iCCM}$ cost per patient contact was USD 18.43. The incremental cost of using the mHealth intervention per child contact for all iCCM conditions was USD 11.35. The incremental cost per treatment of diarrhea, pneumonia, and malaria with mHealth intervention was USD 9.58, USD 10.37 and USD 12.82. Program costs accounted for $67 \%$ of the total, and the largest share was associated with supervision estimated at $36 \%$, followed by supply chain management at $27 \%$. This study has provided valuable information to policy makers on how much it costs to implement iCCM program using mHealth interventions.
\end{abstract}

\section{Introduction}

Despite progress made in reducing child mortality, pneumonia, diarrhea, and malaria remain leading causes of death for children under 5 years of age. ${ }^{1}$ Access to effective and appropriate treatment for sick children is extremely low where it is needed most. ${ }^{2}$ Globally, an estimated 5.9 million children die every year before the age of five; of these deaths, $17 \%$ are due to pneumonia, $8 \%$ due to diarrhea, and $5 \%$ due to malaria. ${ }^{3}$ Notably, sub-Saharan Africa is the region with the highest under five mortality globally. ${ }^{3}$ In Zambia, for example, one in every 13 children will die before their fifth birthday. ${ }^{4}$ Access to prompt and appropriate treatment for these common conditions is therefore crucial in reducing mortality in children under five years. ${ }^{5}$ Integrated community case management (iCCM), implemented by well trained, supplied, and supervised community health workers (CHWs) has the potential to reduce child mortality.

Recognizing this, in 2012, the World Health Organization (WHO) and UNICEF released a "Joint Statement for iCCM" as an equity-focused strategy to improve access to case management, emphasizing important standard practices that should be part of any such programming. ${ }^{6}$ However, iCCM implementation has faced challenges considering the poor health care infrastructure, particularly in low-income countries like Zambia. Frequent stock outs of drugs and commodities, such as malaria rapid diagnostic tests (RDTs) and zinc supplements, are a major challenge to the effectiveness of iCCM. Similarly, studies have considered inadequate supervision of CHWs as one of the most important bottlenecks to effective iCCM implementation. ${ }^{7}$

In order to address supply chain management challenges, mobile health (mHealth) innovations defined as "medical and public health practice supported by mobile devices, such as mobile phones, patient monitoring devices, personal digital assistants (PDAs), and other wireless devices" 1,8 could offer potential solutions that would help move control of stock levels away from central procurement systems to the local level by partly redirecting the immediate monitoring of stocks to the CHWs themselves. Additionally, mHealth has the potential to improve the frequency and quality of supervision and mentoring. ${ }^{8}$ In view of this, mHealth has been incorporated into the field of healthcare in an attempt to address the wide variety of challenges facing low-income countries' health systems, such as skilled worker shortages; a lack of timely reporting for surveillance and diagnostics; poor treatment adherence; and
Correspondence: Godfrey Biemba, National Health Research Authority, Paediatric Centre of Excellence, University Teaching Hospitals, Lusaka, Zambia.

E-mail: gbiemba@gmail.com

Key words: iCCM, malaria, diarrhea, pneumonia, mHealth.

Acknowledgements: The study team would like to acknowledge the following: UNICEF for financial support; Dr. Charlotte Muheki Zikusooka and Cristabel Abewe of HealthNet Consult in Uganda for training the costing data analysis team and developing the costing data collection and analysis tool; the Ministry of Health at all levels for providing data and guidance. In particular, we sincerely thank health facility staff supervisors and CHWs for their cooperation and participation.

Contributions: GB and DH conceptualized and developed the study protocol for the cluster randomized controlled trial, including the costing sub study. They were the Co-Principal Investigators of the study. GB conceptualized the manuscript, contributed to the drafting of the main costing report and interpretation of the costing data; he drafted the first version of the manuscript. BC was the Project Director for the iCCM study. He worked with GB and the team to plan and design the costing work, recruit and train data collectors and supervised the data collection. He read and provided input into the manuscript. DH, KYA, UG, KL, and WML reviewed and had significant input into the manuscript. AM led the costing data collection, analyzed the costing data and drafted the main costing report from which the manuscript was derived. He also reviewed the manuscript. All the authors approved the final version of the manuscript.

Conflict of interest: The authors declare no potential conflict of interest.

Funding: None.

Received for publication: 30 January 2019.

Revision received: 22 August 2019.

Accepted for publication: 17 October 2019.

This work is licensed under a Creative Commons Attribution NonCommercial 4.0 License (CC BY-NC 4.0).

(C) Copyright: the Author(s), 2019

Licensee PAGEPress, Italy

Journal of Public Health in Africa 2019; 10:1039 doi:10.4081/jphia.2019.1039

poor inventory and supply chain management. $^{8}$

Though iCCM has been shown to be an effective strategy for improving access to effective treatment, there are concerns 
about the corresponding cost and added value. ${ }^{9}$ Accordingly, in a resource-constrained setting like Zambia, policy makers or planners are increasingly challenged to justify resource allocation in terms of costs and effects. Arising from this, a comprehensive understanding of $\mathrm{ICCM}$ costs using the mHealth strategy will assist Zambia and other low-income countries that are considering scaling up iCCM using a mHealth strategy to advocate to donors and ministries of finance to allocate sufficient funds for this initiative. ${ }^{10}$

We conducted a cost analysis within a mHealth enhanced iCCM implementation trial. This paper presents the total cost of iCCM and the incremental costs of enhanced supervision and improved drug supply chain management on iCCM implementation using the mHealth strategy.

Trial registration: Study ID No. 4980/A0/04/001/010. ClinicalTrials.gov Identifier: NCT02866097

\section{Materials and Methods}

\section{Study area}

This study was conducted in Chipata and Chadiza Districts of Eastern Province, Zambia. In 2013, Chipata district had 42 health facilities including 30 rural health centers, 7 health posts, 4 urban health centers and 1 level two hospital while Chadiza district had 14 health facilities - 4 health posts, 6 rural health centers, 2 zonal health centers, 1 urban health center and a first level hospital. Data were collected from 40 health facilities (20 iCCM intervention (mHealth) and 20 control sites).

\section{Study design}

The cost analysis was undertaken immediately following the cluster randomized controlled trial (cRCT) that evaluated the effectiveness of a mHealth strategy (intervention) in comparison to the Ministry of Health $(\mathrm{MoH})$ iCCM standard practice (control). The results of the cRCT are yet to be submitted for publication. A cluster was defined as a health facility, including all CHWs in its catchment area that were providing $\mathrm{ICCM}$, and the children under 5 years old with malaria, pneumonia, and diarrhea that they had treated. The primary outcome measure in the cRCT was the proportion of children under five years appropriately treated for malaria, diarrhea, and pneumonia. All clusters sampled for the cRCT were also sampled for the costing analysis.

\section{The iCCM cRCT intervention}

Intervention CHWs were trained to use java-enabled mobile feature phones, loaded with the iCCM Community District Health Management Systems software version 2 (C-DHIS2), Java 2 platform Micro Edition (J2ME) aggregator and tracker applications. ${ }^{11}$ The aggregator application was used to report weekly on disease caseload of malaria, diarrhea, and pneumonia seen and referred by each $\mathrm{CHW}$, and reporting on iCCM commodities using an electronic Report and Requisition Form. The tracker application was used by CHWs to send advance messages to health centers for referred patients, automated messages to health center staff reminding them to conduct monthly mentorship and supervision, and submission of mentorship forms after each mentorship session. Health center staff (CHW supervisors) in the intervention arm were trained to use the C-DHIS2 to receive reports on disease caseloads, medical supplies reports and requisitions, referrals from CHWs, providing feedback to CHWs on cases referred, and to use monthly automated reminders to supervise and mentor. The study was conducted in Chipata and Chadiza districts in Eastern Province of Zambia.

\section{Data collection}

Data collection instruments comprised three questionnaires that were administered through face to face interviews at the district health office from the district iCCM coordinator; at the health facility from the iCCM CHW supervisor; and at the community level from the iCCM trained CHWs. National level data were obtained from the $\mathrm{MoH}$. Data collection instruments were pilot tested and revised before administration in this study. The data collection team included a health economist, who acted as the team leader, and six trained research assistants. These data collectors and supervisors were trained before the pre-test and actual data collection started. Data were collected during August-September 2016 from both the iCCM intervention (mHealth) and control sites with a reference time period of $1^{\text {st }}$ March 2016 - 31 $1^{\text {st }}$ August 2016. From both the intervention and control sites, 40 health facility supervisors and 75 community health workers were interviewed.

\section{Costing methods and perspectives}

In order to calculate the cost of iCCM treatment of malaria, pneumonia and diarrhea in under five years, this study applied the ingredients approach ${ }^{12}$ and adopted the provider's perspective. This included identification of all specific resources used, quantifying them, and evaluating them by attaching either financial and/or economic costs. Data were collected for the baseline year 2016, including prices of medicines, salaries and training. An average exchange rate of ZMW 10.408 per 1 USD in 2016 (www.oanda.com) was used for all USD currency conversions. All research associated costs were excluded.

\section{Recurrent costs}

Recurrent cost items included personnel, management, supervision, operating and maintenance costs (e.g. transport and maintenance of vehicles), overheads (such as water, telephone and electricity), supplies or commodities (such as notebooks, pens, storage bags, gum boots). Supervision costs included costs of staff based at the central, district and health facilities levels that directly supervised the CHWs in the intervention and control sites. In order to compute supervision costs, the percentage time spent on iCCM supervision was allocated to the staff total salary and any other benefits received. Transport and vehicle maintenance included the recurrent costs of maintenance for all the vehicles used in the ICCM program. For the vehicles shared with other programs, an estimate of the proportion of that vehicle's usage was allocated to iCCM.

\section{Capital costs}

Vehicles, cell phones, bicycles and respiratory acute respiratory infection (ARI) timers were the only capital items that were considered. The replacement costs of vehicles were obtained from car dealers in Zambia. Annual replacement costs for vehicles were based on their replacement value at 2016 market prices. Time preference was accounted for by discounting the costs of capital items at a discount rate of 3 percent. ${ }^{13}$ This is consistent with recommendations from literature. ${ }^{14}$ For vehicles, an estimated life of five years was used while for the cell phones, bicycles and respiratory ARI timers an estimated useful life of two years was applied.

\section{Start-up costs}

Start-up costs consisted of training, mHealth software development and cost of equipment (ARI timers, phone and bicycle). Training costs comprised of activities for training supervisors and CHWs on iCCM. For the intervention, the addition of the mHealth strategy required extra training of both CHWs and supervisors in the use of the C-DHSIS2 mobile software. Training cost items were collected from the implementing organization, the Zambian Centre for Applied Health Research and Development Limited (ZCAHRD). mHealth software development costs comprised of the cost of adapting DHIS2 into CDHIS2. 


\section{Dealing with shared costs}

For the computation of the shared overhead costs, we used the proportion of time spent on iCCM program for each overhead expenditure line. In order to obtain the allocation factor, we asked how much time vehicles for instance were used for iCCM or how much time was spent on iCCM. The allocation factor was then applied to the actual overhead expenditure line. For instance, on monthly rentals of about USD $6,666.60,5 \%$ was allocated to iCCM and $2 \%$ was applied to utilities (water, electricity) out of the actual monthly utility bills.

\section{Treatment costs}

Treatment costs included costs of artemisinin-based combination therapy (ACTs), amoxicillin, oral rehydration solution (ORS) and zinc supplements dispensed by CHWs for the treatment of children under five years of age diagnosed with malaria, pneumonia and diarrhea. Drug quantities dispensed were collected from the CHWs patients' registers. Prices of each medicine were sourced from the $\mathrm{MoH}$.

\section{Malaria diagnostic (RDTs) costs}

To compute Malaria diagnostic costs, total number of RDTs used which was obtained from the CHWs patients' registers were multiplied by the unit price of RDT. It should be noted however that data on number of RDTs used by the CHWs were sometimes difficult to obtain due to poor record keeping. Poor record keeping in this case refers to the observation noted on the discrepancy of quantities issued by the health facilities and what was utilized by the CHWs which may have resulted in under reporting of malaria cases diagnosed and treated.

\section{Data entry and analysis}

Data entry began in September 2016, five days after data collection commenced, and continued concurrently with the fieldwork. This offered an advantage because data were consistently quality checked and feedback was given to field teams, thereby improving data quality. All data were first captured on paper-based forms. Once these had been verified as complete and accurate, they were transferred to the ICCM costing Excel tool specifically developed for this study. The costing excel tool automatically calculated the final costs by aggregating all the cost data by cost components (start-up/management costs, program cost, treatment costs). The excel tool also calculated the average unit cost per contact for malaria, pneumonia and diarrhea.

\section{Results}

\section{Study population}

Table 1 summarizes the study population consisting of 8 -iCCM management team at national level, 2 iCCM Coordinators (1 from each of the two study districts), 75 CHWs (38 in intervention and 37 in control sites) and 49 CHW supervisors (29 in intervention and 20 in control site).

\section{Incremental cost per cost centre by study arm}

Table 2 shows incremental cost per cost centre by study arm as start-up, program, and treatment; contributing $32 \%, 67 \%$, and $1 \%$ to the overall incremental cost between intervention and control arms of the study. Training cost contributed the highest to the start-up costs and to the overall incremental cost. Supportive supervision and supply chain management contributed the highest to the incremental costs associated with program costs at $36 \%$ and $27 \%$ of the total incremental cost, respectively. Under supervision, the major cost driver was personnel time at health facilities associated with supervision of CHWs. While, the cost drivers of supply chain management was the costs associated with personnel (pharmacist) at national level and the data bundles provided to the CHWs in the intervention.

\section{Incremental cost (USD) by iCCM disease by study arm}

An analysis of the cost data by iCCM disease category shows that malaria diagnosis and treatment contributed the highest cost to the incremental cost of using mHealth as an intervention to support supervision and supply chain management

Table 1. Description of costing data collection study participants at Chipata and Chadiza Districts, Zambia, 2016.

\begin{tabular}{lccc} 
Participants & Intervention & Control & Total \\
National iCCM management team & & & 8 \\
District iCCM coordinators & & & 2 \\
\hline iCCM CHW supervisors & 29 & 20 & 49 \\
iCCM trained community health workers & 38 & 37 & 75 \\
\hline Total & 67 & 57 & 124 \\
\hline
\end{tabular}

Table 2. Incremental cost per cost centre by study arm in USD, 2016t A016 USD.

\begin{tabular}{|c|c|c|c|c|c|c|}
\hline Cost Centre & & \multicolumn{2}{|c|}{ Study Arm } & $\begin{array}{l}\text { Total } \\
\text { cost }\end{array}$ & $\begin{array}{c}\text { Incremental } \\
\text { cost }\end{array}$ & $\begin{array}{l}\% \text { Contribution to } \\
\text { total incremental cost }\end{array}$ \\
\hline Start-up costs & $\begin{array}{l}\text { Training } \\
\text { Software development for mHealth } \\
\text { Phones, ARI timers, bicycles } \\
\text { Subtotal }\end{array}$ & $\begin{array}{c}92,533 \\
20,000 \\
3,028 \\
115,561\end{array}$ & $\begin{array}{l}51,188 \\
- \\
2,227 \\
53,415\end{array}$ & $\begin{array}{c}143,721 \\
20,000 \\
5,255 \\
168,976\end{array}$ & $\begin{array}{c}41,345 \\
20,000 \\
801 \\
62,146\end{array}$ & $\begin{array}{c}22 \\
10 \\
0 \\
32\end{array}$ \\
\hline Program costs & $\begin{array}{l}\text { Program management } \\
\text { Supervision } \\
\text { Supply chain } \\
\text { Materials (umbrella, notebooks, registers, } \\
\text { gum boots, bags, torches) to CHWs } \\
\text { Vehicles and transport } \\
\text { Personnel } \\
\text { Subtotal }\end{array}$ & $\begin{array}{c}1,869 \\
17,359 \\
5,859 \\
167,678\end{array}$ & $\begin{array}{c}- \\
18,798 \\
331 \\
\\
1,859 \\
14,018 \\
4,741 \\
39,747\end{array}$ & $\begin{array}{c}2,020 \\
107,138 \\
52,562 \\
\\
3,728 \\
31,377 \\
10,600 \\
207,425\end{array}$ & $\begin{array}{c}10 \\
3,341 \\
1,118 \\
127,931\end{array}$ & $\begin{array}{l}0 \\
2 \\
1 \\
67\end{array}$ \\
\hline Treatment costs & Drugs and Supplies & 4,980 & 3,331 & 8,311 & 1,649 & 1 \\
\hline Total costs & & 288,219 & 96,493 & 384,712 & 191,726 & 100 \\
\hline
\end{tabular}


(Table 3). It cost USD 12.82, USD 10.37, and USD 9.58 more to treat each case of malaria, pneumonia, and diarrhea respectively with mHealth enhanced supportive supervision and supply chain management than without mhealth. Overall, it cost USD 11.35 more to treat each iCCM disease with mHealth enhancement than without.

\section{Discussion}

The purpose of this iCCM cost analysis study was to understand the cost implications of providing improved iCCM by enhancing supervision, supply chain management and submission of electronic reports using mHealth strategy in two districts of Zambia. This analysis compares the costs of iCCM using mHealth strategy to the current $\mathrm{MoH}$ standard practice in public health facilities in the two study districts in Zambia. The cost estimates for the iCCM program in these two districts represent an important milestone in obtaining a more comprehensive and accurate reflection of total costs, incremental costs, and cost per iCCM condition. The analysis also calculated the program start-up costs amounting to USD 168,976 which were not included in the computation of incremental cost per iCCM condition.

Higher costs for program and treatment costs items in the intervention sites were constantly observed for each of the expenditure line items compared to the control sites with a resultant overall incremental cost of USD 11.35 per iCCM condition. Within the program costs, highest costs were incurred in the supervision of CHWs by health facility personnel while in the treatment costs, higher costs were incurred in the drugs and supplies expenditure line item. Further, it is worth noting that under program cost there is almost no change in costs for materials to CHWs between intervention and control arms. This is attributed to the fact that the same materials (such as registers, torch, bags, umbrella, boots and $\mathrm{T} /$ shirts) were given to CHWs in both the interventions and control sites. On the other hand, the high supervision costs in the inter- vention sites reflect the relatively greater amount of time spent by health facility staff on supervising CHWs working on iCCM activities in the intervention than in the control sites. The increase in the levels of supervision in the intervention was triggered by CHWs in the submission of electronic report and referrals. The intervention CHWs also attended to more iCCM cases than those in the control. The higher incremental cost of drugs and supplies in treatment costs was attributed to the extra numbers of cases of ICCM conditions seen and the correspondingly larger quantities of the medicine and laboratory supplies consumed in the intervention. Within the intervention sites, confirmed malaria was the most costly iCCM condition followed by diarrhea and pneumonia. A critical look at the drugs and supplies and the number of cases seen as reflected in Tables 2 and 3 show that the intervention arm treated over 3000 more cases for an incremental cost of US1,649.00. It is difficult to fully explain how this apparently small amount of increment covered such a big increase in cases in the intervention. The possible explanation is that the intervention group had greater supervision and mentorship and therefore used drugs and supplies more efficiently, with less wastage.

We also observed a large incremental cost in supply chain. This is attributed to the additional cost of the mobile phones, software programming costs, the cost of data bundles, and the cost of training the CHWs and their supervisors. The overall incremental cost would reduce as the program is scaled up since some of these costs are one off costs.

The total costs reported in this analysis appear to be moderately high. However, it should be noted that, while efforts were made in our cost analysis methodology to discount costs associated with research activities; for example, staff costs by the investigators, not all costs could easily be discounted because they were intricately associated with both research and program implementation. A good example was the cost associated with the time spent on the project by the Project Director who coordi- nated both program and research activities. This limitation could have over-estimated the costs. The incremental cost of USD 11.35 per iCCM condition treated appears to be fairly large. However, we were unable to find any literature on the incremental cost of mHealth in ICCM and hence it may be difficult to objectively say this figure is high or low since we do not have any regional or national market figures to compare with.

The cost per iCCM case treated ranged from USD 19.53 to USD 16.97 for mHealth enhanced treatment of malaria and pneumonia, respectively, and from USD 9.08 to USD 6.60 for non-mHealth enhanced treatment of diarrhea and pneumonia, respectively. The figures for the cost of nonmHealth enhanced treatment of iCCM conditions are comparable to figures reported in literature within the African region, reporting a cost range of USD 2.44 to 13.71 per diarrhea case treated; USD 2.17 to USD 17.54 per for malaria (excluding rapid diagnostic tests); USD 1.70 to USD 12.94 per pneumonia treated. ${ }^{10} \mathrm{We}$ do note however that, according to the analysis by Collins, the costs for Zambia in 2011 were USD 3.60 and 3.56 per diarrhea and pneumonia treatment, respectively. The difference between our analysis and Collins' results could be due to changes in unit costs over time.

The overall costs of iCCM implementation should become lower in relative terms over time if the program is scaled up and achieves economies of scale. There are also more opportunities for minimizing costs while maintaining good support for the CHWs so that the availability and quality of supervision of iCCM is maintained. For instance, costs can be reduced by integrating activities such as combining supervision with outreach visits where extra curative services are provided by the supervisor during the visit or focusing on all community health services rather than just the iCCM program.

\section{Limitations}

When interpreting the results of this iCCM costing analysis, a number of methodological limitations should be considered.

Table 3. Incremental cost in USD by iCCM disease by study arm, 2016.

\begin{tabular}{|c|c|c|c|c|c|c|c|}
\hline \multirow[t]{2}{*}{ iCCM cases } & \multicolumn{3}{|c|}{ Intervention } & \multicolumn{3}{|c|}{ Control } & \multirow{2}{*}{$\begin{array}{c}\text { Incremental } \\
\text { Cost/Case } \\
\text { Cost }\end{array}$} \\
\hline & Total \# of Cases & Total Cost & Cost per Case & Total \# of Cases & Total Cost & Cost per Case & \\
\hline Diarrhea & 1,689 & $31,514.90$ & 18.66 & 1,063 & $9,648.00$ & 9.08 & 9.58 \\
\hline Pneumonia & 3,441 & $58,390.10$ & 16.97 & 2,339 & $15,439.00$ & 6.60 & 10.37 \\
\hline Malaria (all confirmed) & 4,245 & $82,889.71$ & 19.53 & 2,686 & $18,026.00$ & 6.71 & 12.82 \\
\hline Total & 9,375 & $172,794.71$ & 18.43 & 6,088 & $43,113.00$ & 7.08 & 11.35 \\
\hline
\end{tabular}




\section{References}

Personnel costs were estimated based on activities as reported by the staff involved. These relied on respondents' recall of the proportion of staff time devoted to the iCCM activities. This could have resulted in overor under-estimation of the actual costs. In addition, as noted under the discussion section, while efforts were made to separate research costs from purely iCCM implementation costs, complete separation was not possible. Hence, some of the costs included in this analysis may have included research costs, leading to a possible over estimate of the costs. Notwithstanding these limitations, this analysis presents findings that at best lay out to decision makers a rigorously estimated cost of the iCCM program using mHealth strategy.

\section{Conclusions}

This cost analysis study of ICCM in Chipata and Chadiza, rural districts of Zambia, has provided practical, applicable information on the costs associated with implementing an iCCM program using mHealth interventions. The study shows that enhancing iCCM using mobile health technology was associated with an incremental cost of USD 11.50 per child contact. Over two thirds of this cost was attributable to program costs; $36 \%$ of which was associated with supervision. If the program is to be scaled up, the incremental cost per child contact should become lower, as all costs associated with research activities would not be included. Further research is needed to evaluate the added long-term value of mHealth interventions on the health outcomes that iCCM addresses to determine the incremental cost-effectiveness of mHealth enhancements of iCCM.
1. WHO (2011). Child mortality. Millennium Development Goal (MDG). Available from: http://www.who.int/ $\mathrm{pmnch} / \mathrm{media} / \mathrm{press}$ materials/fs/fs_md g4 childmortality/en/

2. George A, Rodríguez DC, Rasanathan $\mathrm{K}$, et al. iCCM policy analysis: Strategic contributions to understanding its character, design and scale up in subSaharan Africa. Health Policy Plan 2015;30,:ii3-ii11.

3. UNICEF (2015). Levels and trends in child mortality. Report on estimates developed by the UN Inter-agency Group for Child Mortality Estimation. https:/www.unicef.org/media/files/IG ME_Report_Final2.pdf

4. Zambia Demographic and Health Survey (ZDHS) 2013-14, Central Statistical Office (CSO), Ministry of Health (MOH) [Zambia], ICF International, Rockville, Maryland, USA: Central Statistical Office, Ministry of Health, and ICF International.

5. Nonvignon J, Chinbuah MA, Gyapong $\mathrm{M}$, et al. Is home management of fevers a cost-effective way of reducing underfive mortality in Africa? The case of a rural Ghanaian District. Trop Med Int Health 2012;17:951-7.

6. Diaz T, Aboubaker S, Young M. Current scientific evidence for integrated community case management (iCCM) in Africa: Findings from the iCCM Evidence Symposium. J Global Health 2014;4:020101.

7. Hamer DH, Marsh DR, Peterson S, Pagnoni F. Integrated community case management: next steps in addressing the implementation research agenda.
Am J Trop Med Hyg 2012;87:151-3.

8. Marshall C, Lewis D, Whittaker M . mHealth technologies in developing countries, 2013: a feasibility assessment and a proposed framework. https:/ /www.researchgate.net/publication/264 205169_Strengthening_health_systems_in_mHealth_technologies_in_dev eloping_countries_a_feasibility_assessment_and_a_proposed_framework Accessed: July 23, 2018.

9. Collins D, Jarrah Z, Gilmartin C, Saya $\mathrm{U}$. The costs of integrated community case management (iCCM) programs: A multi-country analysis. J Global Health 2014;4:020407.

10. Nefdt R, Ribaira E, Diallo K. Costing commodity and human resource needs for integrated community case management in the differing community health strategies of Ethiopia, Kenya and Zambia. Ethiop Med J 2014;52:137-49.

11. Biemba G, Chiluba B, Yeboah-Antwi $\mathrm{K}$, et al. Mobile-Based Community Health Management Information System for Community Health Workers and Their Supervisors in 2 Districts of Zambia. Glob Health Sci Pract 2017;5: 486-94.

12. Drummond FM, Sculpher JM, Torrance WG, et al.. Methods for The Economic Evaluation Of Health Care Programmes, Oxford University Press. 2005.

13. Drummond MF, Stoddart GL, Torrance GW. Methods for Economic Evaluation of Health Care Programmes, 1997; 2nd ed, Oxford: Oxford University Press.

14. Walker D, Kumaranayake L. Allowing for differential timing in cost analyses discounting and annualization. Health Policy Plann 2002;17:112-8. 